\title{
Morte e morrer: Caminhos utilizados por docentes de enfermagem na formação
}

\section{acadêmica}

\author{
Death and dying: Paths used by nursing teachers in academic training \\ Muerte y morir: Caminos de los profesores de enfermería en la formación académica
}

\author{
Maria Luiza Azevedo dos Reis \\ ORCID: https://orcid.org/0000-0001-8830-0785 \\ Universidade Federal de Campina Grande, Brasil \\ E-mail: 1uizareis21@gmail.com \\ Olavo Mauricio de Souza Neto \\ ORCID: https://orcid.org/0000-0003-0318-7192 \\ Universidade Federal de Campina Grande, Brasil \\ E-mail: olavomauricio128@gmail.com \\ Josefa Eucliza Casado Freires da Silva \\ ORCID: https://orcid.org/0000-0003-1066-0574 \\ Universidade Federal de Campina Grande, Brasil \\ E-mail: euclisa14@hotmail.com \\ Walkerlane Adelaide Damasceno Silva \\ ORCID: https://orcid.org/0000-0003-4263-5509 \\ Universidade Federal de Campina Grande, Brasil \\ E-mail: walkerlane25@gmail.com \\ Maria Aparecida Martins \\ ORCID: https://orcid.org/0000-0002-8204-6584 \\ Universidade Federal de Campina Grande, Brasil \\ E-mail: aparecidamarthyns@gmail.com \\ Glenda Agra \\ ORCID: https://orcid.org/0000-0002-7628-9029 \\ Universidade Federal de Campina Grande, Brasil \\ E-mail: g.agra@yahoo.com.br
}

\begin{abstract}
Resumo
Introdução: A morte não é somente um fato biológico do desenvolvimento humano, mas um processo construído socialmente, que não se distingue das outras dimensões do universo das relações sociais Objetivo: Investigar os caminhos utilizados pelos docentes de enfermagem acerca da temática morte e morrer na formação acadêmica. Metodologia: Trata-se de um estudo transversal com abordagem quantitativa realizado com docentes de um curso de Bacharelado em Enfermagem de uma universidade pública da Paraíba. Os dados foram analisados com estatística descritiva, analisados à luz da literatura pertinente. Resultados: De forma geral, pode-se observar que a maioria dos docentes de enfermagem abordam a temática morte e morrer na formação acadêmica, de forma transversal, uma vez que a grade curricular contempla disciplinas que abrangem os processos de nascer, viver, morrer e luto com vistas à qualidade de vida e dignidade humana. Conclusão: Se faz necessário conversar sobre a morte, trazê-la para dentro da academia, abrindo espaços de educação para morte, seja no ensino, na pesquisa ou na extensão. O importante é trazer à tona reflexões, discussões e ações que aproximem os acadêmicos do fenômeno morte do mesmo modo que experienciam o fenômeno do nascimento, desenvolvimento e envelhecimento do ser humano.
\end{abstract}

Palavras chaves: Educação em enfermagem; Docentes de enfermagem; Morte.

\begin{abstract}
Introduction: Death is not only a biological fact of human development, but a socially constructed process, which is indistinguishable from other dimensions of the universe of social relations Objective: To investigate the paths used by nursing professors on the theme of death and dying in training academic. Methodology: This is a cross-sectional study with a quantitative approach carried out with professors of a Bachelor's Degree in Nursing at a public university in Paraíba. Data were analyzed using descriptive statistics, analyzed in the light of relevant literature. Results: In general, it can be observed that most nursing professors address the theme of death and dying in academic training, in a crosssectional way, since the curriculum includes subjects that cover the processes of being born, living, dying and I struggle with a view to quality of life and human dignity. Conclusion: It is necessary to talk about death, bring it into the academy, opening spaces for education for death, whether in teaching, research or extension. The important thing is to bring up reflections, discussions and actions that bring academics closer to the phenomenon of death in the same way they experience the phenomenon of birth, development and aging of human beings.
\end{abstract}


Keywords: Nursing education; Nursing professors; Death.

\section{Resumen}

Introducción: La muerte no es solo un hecho biológico del desarrollo humano, sino un proceso socialmente construido, indistinguible de otras dimensiones del universo de las relaciones sociales Objetivo: Investigar los caminos recorridos por los profesores de enfermería sobre el tema de la muerte y el morir en la educación académico. Metodología: Se trata de un estudio transversal con enfoque cuantitativo realizado con profesores de la Licenciatura en Enfermería de una universidad pública de Paraíba. Los datos se analizaron mediante estadística descriptiva, analizados a la luz de la literatura relevante. Resultados: En general, se puede observar que la mayoría de los profesores de enfermería abordan el tema de la muerte y el morir en la formación académica, de manera transversal, ya que el currículo incluye asignaturas que abarcan los procesos de nacer, vivir, morir y lucho. con miras a la calidad de vida y la dignidad humana. Conclusión: Es necesario hablar de la muerte, llevarla a la academia, abriendo espacios para la educación para la muerte, ya sea en la docencia, la investigación o la extensión. Lo importante es plantear reflexiones, discusiones y acciones que acerquen a los académicos al fenómeno de la muerte de la misma manera que viven el fenómeno del nacimiento, desarrollo y envejecimiento de los seres humanos.

Palabras clave: Educación en enfermería; Profesores de enfermería; Muerte.

\section{Introdução}

A morte é um tema visto sob diferentes dimensões, que não permite afirmar verdades absolutas, pois, quando abordado, desperta curiosidade, provoca desconforto e vem sempre acompanhada de muitas perguntas para as quais se encontra a incontestável resposta de que o morrer é inevitável, intrínseco à vida e representa a certeza de que a todo nascimento associa-se um momento de fim (Carneiro \& Andrade, 2018).

Para Moura et al. (2018), a morte não é somente um fato biológico do desenvolvimento humano, mas um processo construído socialmente, que não se distingue das outras dimensões do universo das relações sociais. Assim, a morte está presente no cotidiano do ser humano, independentemente de suas causas ou formas.

É importante lembrar que, dentre todos os seres humanos que precisam conviver com os sentimentos provocados pela morte, os profissionais da área da saúde se encontram mais suscetíveis, pois no cenário das instituições hospitalares a morte está constantemente presente, motivo pelo qual é tema relevante, porém de difícil abordagem reflexiva, principalmente na prática de cuidado da enfermagem (Nunes, Araújo \& Silva, 2016).

Por conviverem mais diretamente e mais tempo com os pacientes, os profissionais de enfermagem estão mais expostos, no entanto, isso não significa que estejam mais preparados (Nunes et al., 2016; Nunes \& Santos, 2017; Prado et al., 2017; Lima \& Nietsche, 2016). Pois, desde a sua formação, o enfermeiro se sente compromissado com a vida e é para preservação desta que deverá se sentir capacitado. Sua formação acadêmica está fundamentada na cura e nela está a sua maior gratificação. Assim, quando em seu cotidiano de trabalho necessita lidar com a morte, em geral, sente-se despreparado, e tende a se afastar dela (Nunes et al., 2016; Nunes \& Santos, 2017; Prado et al., 2017).

Justamente por isso, a questão da morte e do morrer deveria ser objeto privilegiado de sua formação profissional, fosse para oferecer capacidade técnica adequada ou por uma questão de segurança à sua saúde mental (Kovács, 2004; Nunes et al., 2016; Nunes \& Santos, 2017).

A experiência profissional nos mostra que a abordagem do processo de morte e morrer tem sido feita de maneira rápida e superficial durante a formação do enfermeiro, não havendo momentos formalmente estipulados no currículo para que a discussão sobre essa temática aconteça. Contrariamente, percebe-se ainda que a morte é abordada de maneira negativa através de seu oposto, ou seja, a manutenção do corpo vivo pelo emprego de todos os esforços profissionais e tecnológicos possíveis. Essa situação gera um paradoxo para os profissionais enfermeiros que cuidam de pessoas que estão passando pelo processo de finitude humana, pois oscilam entre necessidades igualmente prementes de manter a pessoa viva, a todo custo, e ao mesmo tempo, ajudá-la a morrer de maneira mais tranquila e digna possível (Bandeira et al., 2014; Prado et al., 2017; Carneiro \& Andrade, 2018; Lima \& Nietsche, 2016; Nunes \& Santos, 2017; Moura, 2018; Silva \& Melo, 2018; Praxedes et al., 2018). 
Tal fato tem contribuído para que haja, não apenas uma lacuna na formação acadêmica, como também a negação da morte como parte integrante da vida, gerando consequências, profissionais pouco aptos a prestar cuidados de maneira abrangente às pessoas que vivenciam a finitude, bem como suas famílias. Este despreparo para o cuidado a essas pessoas é sentido pelos enfermeiros e pode favorecer a fuga de uma situação de confronto com os seus próprios medos e angústias, levando-os a limitarem-se, na maioria das vezes, a cuidar do corpo que está morrendo, e não do ser humano que morre (Prado et al., 2017; Carneiro \& Andrade, 2018; Lima \& Nietsche, 2016; Nunes \& Santos, 2017; Moura, 2018; Silva \& Melo, 2018; Praxedes et al., 2018).

A partir deste contexto, torna-se necessário refletir também sobre as condições que os docentes, responsáveis por essa formação, têm para estarem abordando essa temática com os estudantes. Estarão eles preparados para esse enfrentamento e, de maneira concomitante, para o ensino desse processo na sua prática docente?

Espera-se contribuir para a discussão acerca da necessidade de preparo formal de enfermeiros para o desempenho do cuidado de enfermagem prestado a pessoas diante do processo de finitude, bem como o preparo do docente para possibilitar e propiciar condições para que essa discussão possa ocorrer em disciplinas que atua, uma vez que ambas as dimensões estão intrinsecamente associadas.

Diante deste contexto e tomando como referência a formação dos profissionais de enfermagem, surge a seguinte pergunta: como os docentes de enfermagem abordam o processo de morte e morrer durante a graduação?

Diante desta questão, entendemos a importância deste estudo para obtenção de algumas respostas, com a finalidade de identificar como os docentes de enfermagem abordam a temática do processo de morte e morrer durante as disciplinas teóricas e práticas na graduação em enfermagem.

Nesta perspectiva, o objetivo geral desta pesquisa é investigar os caminhos utilizados pelos docentes de enfermagem acerca da temática morte e morrer na formação acadêmica.

\section{Metodologia}

Trata-se de um estudo transversal de natureza quantitativa realizado no Centro de Educação em Saúde (CES) da Universidade Federal de Campina Grande (campus Cuité - PB). De acordo com Pereira et al. (2018), a pesquisa quantitativa é um dos métodos estatísticos no qual, faz-se a coleta de dados quantitativos ou numéricos através do uso de medições de grandezas, adquirindo por meio da metrologia, números com suas respectivas unidades. Nesse sentido, os métodos quantitativos geram conjuntos ou massas de dados que podem ser analisados por meio de técnicas matemáticas como é o caso das porcentagens, estatísticas e probabilidade, dentre outras possibilidades de trabalho com o método quantitativo.

A população para este estudo foi composta por docentes de enfermagem e a amostra foi de docentes de enfermagem da Universidade Federal de Campina Grande, do Centro de Educação em Saúde (campus Cuité - PB). Os critérios de inclusão para seleção da amostra foram: ser docente de enfermagem da Universidade Federal de Campina Grande do Centro de Educação em Saúde, campus Cuité- PB, do quadro permanente de pessoal, independente do regime de trabalho. Os critérios de exclusão foram: docentes que estivessem afastados para capacitação docente e/ou afastados por licença saúde e/ou maternidade.

O instrumento foi um questionário semiestruturado, baseado no estudo de Bellator et al. (2007), composto por dados de identificação dos participantes da pesquisa (docentes de enfermagem) e perguntas subjetivas que visavam atender aos objetivos do estudo. A coleta de dados foi realizada no período de dezembro de 2020 a março de 2021, após a aprovação do projeto pelo Comitê de Ética em Pesquisas, sob o CAAE 16375119.4.0000.5182 e Parecer nº 3.620.975.

$\mathrm{O}$ sigilo, e a desistência em qualquer momento da pesquisa foram garantidos, mediante a assinatura de um Termo de Consentimento Livre e Esclarecido (TCLE). Os critérios utilizados obedeceram à Resolução no 466/2012 do Conselho 
Nacional de Saúde que norteia pesquisas envolvendo seres humanos. A pesquisa foi realizada após a anuência do diretor Centro de Educação e Saúde, onde os docentes exercem atividades laborais e posteriormente, autorização do Comitê de Ética em Pesquisa (Brasil, 2012). Todas essas exigências foram devidamente respeitadas durante a operacionalização desta pesquisa, assim como as premissas observadas na Resolução $n^{\circ}$ 564//2017 do Conselho Federal de Enfermagem, que trata da reformulação do Código de Ética Profissional (Cofen, 2017).

Após o convite para participar da pesquisa e concordância em fazer parte do estudo os participantes foram esclarecidos quanto aos objetivos do estudo. As respostas de cada questão foram registradas em tabelas, usando estatística descritiva inferencial e os dados foram analisados à luz da literatura.

\section{Resultados}

Participaram da pesquisa 21 (95,5\%) enfermeiros docentes do Curso de Bacharelado em Enfermagem; destes, 19 eram mulheres (90,7\%) e dois, homens (9,5\%); com idade média de 38 anos. A pesquisa foi realizada em um campus universitário público situado na região do Curimataú no Estado da Paraíba (PB), localizado no município de Cuité - PB.

O Curso de Bacharelado em Enfermagem possui competências para atender às demandas de saúde do indivíduo, família e comunidade, na perspectiva da vigilância em saúde, além de possuir visão crítica, reflexiva e criativa do contexto social, cultural, político e econômico, de modo a possibilitar sua participação em ações transformadoras do cenário no qual esteja inserido, conforme a aquisição de habilidades e atitudes oriundas da sua formação.

Os resultados deste estudo foram distribuídos em tabelas, para melhor organização e entendimento. Abaixo, segue a Tabela 1, que apresenta a distribuição dos dados profissionais dos docentes participantes da pesquisa.

Tabela 1 - Distribuição dos dados profissionais dos docentes, Cuité - PB, 2021.

\begin{tabular}{|c|c|c|c|c|c|}
\hline $\begin{array}{l}\text { Atuação na } \\
\text { enfermagem }\end{array}$ & $\begin{array}{c}\text { Atuação } \\
\text { na } \\
\text { docência }\end{array}$ & $\begin{array}{c}\text { Área } \\
\text { hospitalar }\end{array}$ & $\begin{array}{l}\text { Atuação na } \\
\text { assistência } \\
\text { hospitalar }\end{array}$ & Disciplina & Semestre \\
\hline 7 anos & 5 anos & Sim & $\begin{array}{l}\text { Clínica médica } \\
\text { e cirúrgica }\end{array}$ & $\begin{array}{c}\text { Ética, Legislação e } \\
\text { Bioética em } \\
\text { Enfermagem; Bases } \\
\text { Teóricas de Enfermagem } \\
\text { em Saúde Coletiva III }\end{array}$ & $5^{\circ}$ \\
\hline 9 anos & 7 anos & Sim & UTI & $\begin{array}{l}\text { Farmacologia Aplicada } \\
\text { em Enfermagem; } \\
\text { Estágio Supervisionado } \\
\text { em Enfermagem II }\end{array}$ & $4^{\circ}$ e $10^{\circ}$ \\
\hline 10 anos & 8 anos & Não & - & $\begin{array}{c}\text { Enfermagem em } \\
\text { Cuidados Críticos; } \\
\text { Enfermagem na Saúde } \\
\text { do Homem; Bases } \\
\text { Teóricas de Enfermagem } \\
\text { em Cirúrgica I }\end{array}$ & $\begin{array}{c}4^{\circ}, 7^{\circ} \mathrm{e} \\
8^{\circ}\end{array}$ \\
\hline 10 anos & 5 anos & Não & - & $\begin{array}{c}\text { Educação em Saúde; } \\
\text { Estágio Supervisionado } \\
\text { em Enfermagem I; } \\
\text { Trabalho de Conclusão } \\
\text { de Curso I }\end{array}$ & $6^{\circ}, 8^{\circ}$ e $9^{\circ}$ \\
\hline 10 anos & 8 anos & Sim & $\begin{array}{l}\text { Atendimento } \\
\text { Pré-Hospitalar, } \\
\text { Pediatria }\end{array}$ & $\begin{array}{l}\text { Bases Teóricas de } \\
\text { Enfermagem em } \\
\text { Semiologia e } \\
\text { Semiotécnica I }\end{array}$ & $3^{\circ}$ \\
\hline 10 anos & 4 anos & Sim & $\mathrm{CCIH}$ & $\begin{array}{c}\text { Embriologia } \\
\text { Humana/Histologia/Para }\end{array}$ & $\begin{array}{l}1^{\mathrm{o}}, 2^{\mathrm{o}}, 3^{\mathrm{o}} \\
\mathrm{e} 2^{\mathrm{o}}\end{array}$ \\
\hline
\end{tabular}




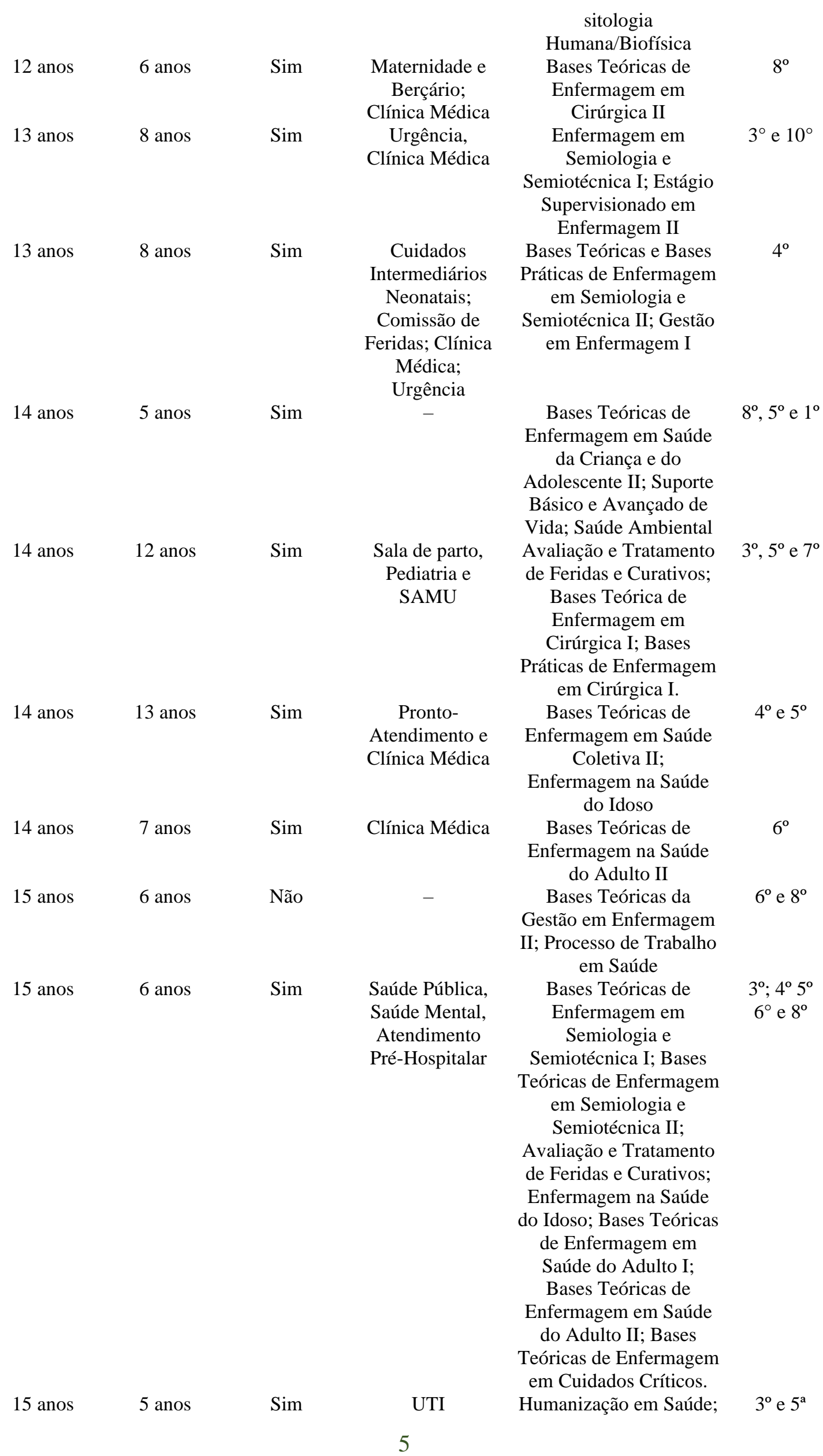




\begin{tabular}{|c|c|c|c|c|c|}
\hline & & & & $\begin{array}{c}\text { Bases Teóricas de } \\
\text { Enfermagem na Saúde } \\
\text { da Mulher. }\end{array}$ & \\
\hline 16 anos & 12 anos & Sim & $\begin{array}{c}\text { Emergência } \\
\text { Pediátrica }\end{array}$ & $\begin{array}{l}\text { Práticas Integrativas e } \\
\text { Complementares em } \\
\text { Saúde; Bases Teóricas } \\
\text { de Enfermagem em } \\
\text { Psiquiatria }\end{array}$ & $7^{\circ}$ \\
\hline 16 anos & 15 anos & Sim & Infectologia & $\begin{array}{c}\text { Enfermagem em } \\
\text { Neonatologia; Bases } \\
\text { Teóricas de Enfermagem } \\
\text { na Saúde da Criança e } \\
\text { Adolescente I }\end{array}$ & $6^{\circ}$ e $7^{\circ}$ \\
\hline 16 anos & 16 anos & Sim & $\begin{array}{l}\text { Clínica } \\
\text { Obstétrica }\end{array}$ & $\begin{array}{c}\text { Bases Teóricas de } \\
\text { Enfermagem em } \\
\text { Obstetrícia; Saúde } \\
\text { Coletiva I }\end{array}$ & $3^{\circ}$ e $6^{\circ}$ \\
\hline 17 anos & 10 anos & Sim & $\begin{array}{l}\text { Saúde mental e } \\
\text { Psiquiatria, } \\
\text { Bloco Cirúrgico }\end{array}$ & $\begin{array}{c}\text { Saúde Mental; Estágio } \\
\text { Supervisionado em } \\
\text { Enfermagem I }\end{array}$ & $6^{\circ}$ e $9^{\circ}$ \\
\hline 29 anos & 18 anos & Sim & UTI e Urgência & $\begin{array}{l}\text { Bases Teóricas de } \\
\text { Enfermagem em } \\
\text { Cuidados Críticos; } \\
\text { Primeiros Socorros. }\end{array}$ & $6^{\circ}$ \\
\hline
\end{tabular}

Fonte: Dados da Pesquisa (2021).

A seguir, apresenta-se a Tabela 2, que destaca a distribuição dos sentimentos dos docentes da pesquisa quando relacionados à temática morte e morrer.

Tabela 2 - Distribuição dos sentimentos dos docentes, Cuité - PB, 2021.

\begin{tabular}{ccc}
\hline SENTIMENTOS & $\mathbf{N}^{\circ}$ & $\mathbf{F}(\%)$ \\
Aceitação & 12 & $57,1 \%$ \\
Medo & 11 & $52,3 \%$ \\
Pavor & 10 & $47,6 \%$ \\
Angústia & 8 & $38 \%$ \\
Tristeza & 8 & $38 \%$ \\
Saudade & 4 & $19 \%$ \\
Sofrimento & 4 & $19 \%$ \\
Raiva & 2 & $9,5 \%$ \\
Negação & 1 & $4,7 \%$ \\
\hline
\end{tabular}

Fonte: Dados da Pesquisa (2021).

Abaixo, apresenta-se o Gráfico 1, que destaca a distribuição dos resultados relativos ao processo de ensinoaprendizagem dos docentes sobre a temática morte e morrer no Curso de Bacharelado em Enfermagem. 
Gráfico 1 - Distribuição dos dados sobre o processo de ensino/aprendizagem dos docentes sobre a temática morte/morrer dos docentes, Cuité - PB, 2021.

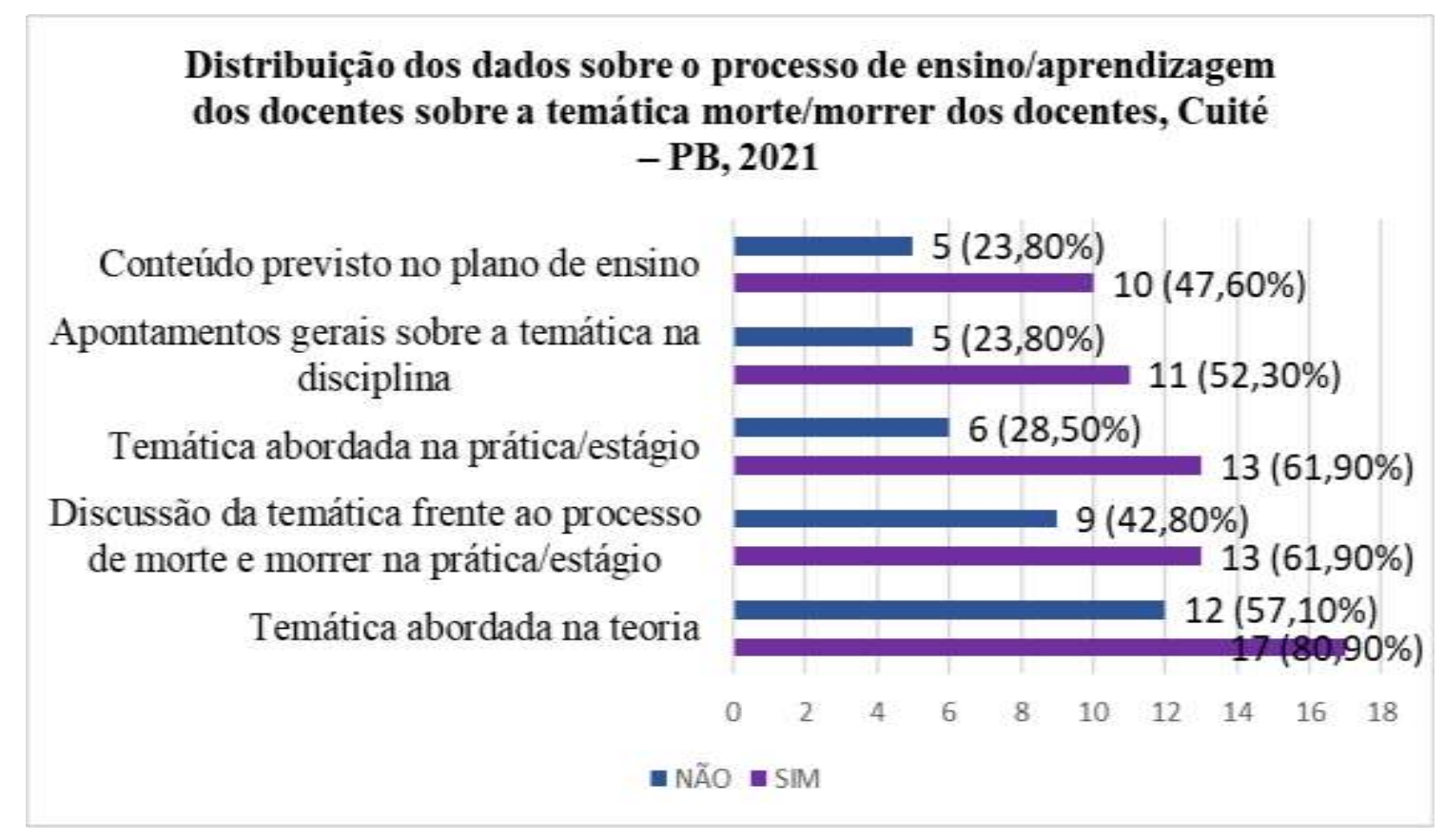

Fonte: Dados da Pesquisa (2021).

A seguir, está a Tabela 4, que descreve as estratégias educativas e aspectos enfatizados pelos docentes durante o processo de ensino-aprendizagem sobre a temática morte e morrer.

Tabela 4 - Distribuição das estratégias educativas e aspectos enfatizados pelos docentes durante o processo de ensinoaprendizagem sobre a temática morte e morrer, Cuité - PB, 2021.

\begin{tabular}{ccc}
\hline $\begin{array}{c}\text { ESTRATÉGIAS } \\
\text { EDUCATIVAS }\end{array}$ & $\mathbf{n}$ & $\mathbf{f}(\boldsymbol{\%})$ \\
Aula expositiva-dialogada & 9 & $42,8 \%$ \\
Acompanhar os acadêmicos & & \\
durante os cuidados de & 5 & $23,8 \%$ \\
enfermagem à pessoas em & & \\
processo de morte e morrer e seus & & \\
$\quad$ familiares & 5 & $23,8 \%$ \\
Trabalhos em grupos, seminários & 4 & $19 \%$ \\
Projeção de filmes, vídeos & 2 & $9,5 \%$ \\
Leitura de textos, livros & 2 & $9,5 \%$ \\
Estudos de casos & 2 & $9,5 \%$ \\
Visitas técnicas & & $\mathbf{F}(\%)$ \\
ASPECTOS ENFATIZADOS - & $\mathbf{N}^{\circ}$ & $61,9 \%$ \\
ENSINO/APRENDIZAGEM & 13 &
\end{tabular}


paciente e à família durante o

processo de morte e morrer

A doença que causou a morte

Os sentimentos dos acadêmicos e

da equipe de saúde/enfermagem

diante da situação de morte do

paciente

Condutas administrativas a serem

tomadas no caso

Como proceder com o preparo do corpo

Fonte: Dados da Pesquisa (2021).

A Tabela 5 que está logo abaixo, descreve os cuidados de enfermagem realizados pelos alunos em pacientes em processo de morte e morrer.

Tabela 5 - Distribuição dos cuidados de enfermagem realizados pelos alunos a pacientes em processo de morte e morrer, Cuité - PB, 2021.

\begin{tabular}{ccc}
\hline CUIDADOS DE & $\mathbf{N}^{\circ}$ & F (\%) \\
ENFERMAGEM NO & & \\
ÂMBITO DA SAE & 10 & $47,6 \%$ \\
Cuidados espirituais & 5 & $23,8 \%$ \\
Cuidados psíquicos & 2 & $9,5 \%$ \\
Cuidados sociais & 1 & $4,7 \%$ \\
Cuidados físicos & 1 & \\
\hline
\end{tabular}

Fonte: Dados da Pesquisa (2021).

\section{Discussão}

No que se refere à caracterização dos participantes da pesquisa, observou-se que as mulheres representam a maioria $(90,7 \%)$ dos docentes do campus universitário lócus da pesquisa. A explicação dada para esse contingente de mulheres na área educacional é devida à "vocação" que esteve vinculada à expertise feminina, já que ensinar estava relacionado com a continuidade do cuidado e, portanto, com a obrigação da mulher na sociedade patriarcal. Os dados da Pesquisa Nacional por Amostra de Domicílio (PNAD) de 2004 corroboram essa ideia - 78,2\% dos profissionais da educação são mulheres (Andrade, 2015).

O estudo das relações de gênero na enfermagem perpassa a história do desenvolvimento da profissão. As atividades de cuidado que caracterizam o trabalho das enfermeiras passam pelo processo de profissionalização, com respaldo na legislação, exigindo-se, assim, a formação de nível superior. A história mostra que, em 1949, foi promulgada a Lei que legislava sobre o ensino de enfermagem, caracterizado pelo curso de graduação e o de auxiliar. Entretanto, foi somente com a promulgação da Lei 4.024, pós-Lei de Diretrizes e Bases - LDB de 1961, que o curso passou a ser de nível superior (Pereira \& Ramos, 2006). 
No que diz respeito aos resultados da Tabela 1, observou-se uma média de 14 anos de atuação na assistência de enfermagem, destacando-se as áreas da clínica médica e da UTI; já na docência, a média de atuação foi de 8 anos. As disciplinas mencionadas pelos docentes vão desde componentes curriculares básicos obrigatórios (por ex. Embriologia, Parasitologia, Farmacologia dentre outros) e específicos teórico-práticos (por ex. Bases Teóricas e Práticas na Saúde da Criança e Adolescente, da Mulher, do Adulto, do Idoso dentre outros) e componentes específicos práticos (por ex. Estágio Supervisionado I e II). Vale ressaltar que os componentes curriculares ministrados pelos docentes participantes da pesquisa distribuem-se desde o início do curso e vão até o $10^{\circ}$ período, perfazendo um quantitativo de cinco anos de graduação.

Observa-se, dessa forma, que os docentes de enfermagem se amparam na Resolução do Conselho Nacional de Saúde (CNS) n 573 de 31 de janeiro de 2018, que trata das Recomendações do Conselho Nacional de Saúde à proposta das Diretrizes Curriculares Nacionais (DCN) do curso de graduação Bacharelado em Enfermagem ao estabelecer no Projeto Pedagógico do Curso (PPC), as capacidades profissionais que mobilizem conhecimentos, saberes, habilidades e atitudes, que resultem em aptidão para atuação profissional em sistema de produção de serviços de saúde. Nesse contexto, observou-se que os docentes, por meio dos componentes curriculares básicos e específicos disponibilizam vivências na Rede de Atenção à Saúde (RAS), atividades consideradas essenciais para a saúde de pessoas, grupos sociais e populações (Brasil, 2018).

No que se concerne aos resultados da Tabela 2, que trata dos sentimentos dos docentes em relação à temática morte e morrer, observou-se que o sentimento de aceitação $(57,1 \%)$ foi o mais destacado, seguido do medo $(52,3 \%)$ e do pavor $(47,6 \%)$. Sobre este aspecto, vale ressaltar que os profissionais de Enfermagem podem ter concepções diferentes em relação à morte e uma delas é a percepção da morte como processo natural da vida, como a maioria dos docentes referiram a aceitação como sentimento relacionado à temática, ou seja, eles compreendem que a morte faz parte do processo de vida do ser humano, que é um evento natural do ponto de vista biológico, que faz parte da condição humana e, portanto, é a marca existencial de todas as pessoas (Kovács, 2008).

Porém, ainda que a morte e o morrer sejam fenômenos inevitáveis, refletir sobre a finitude humana é algo desafiador e suscita sentimentos diversos, que vão desde a aceitação até sentimentos mais angustiantes, tais como medo, pavor dentre outros (Barbosa, Francisco \& Efken, 2008), assim como pode-se observar que parte dos docentes desta pesquisa referiram sentimentos de medo $(52,3 \%)$ e pavor (47,6\%). Nesse sentido, Kovács (2008), ao referir sobre os sentimentos relacionados à morte, afirma que ao presenciar o falecimento de outrem, algumas pessoas apresentam sentimentos diversos, dentre eles, o medo e o pavor.

Nesta perspectiva, vale ressaltar que alguns estudos explicam e consideram o medo da morte como fator multidimensional (Collet \& Lester, 1969; Hoelter, 1979; Conte, Weiner \& Plutchik, 1982), a saber: medo de morrer; dos mortos; de ser destruído; da perda de pessoas significativas; do desconhecido; da morte consciente; do corpo após a morte; da morte prematura (Hoelter, 1979).

Esta multidimensionalidade está relacionada a algumas variáveis, dentre elas, à exposição à morte do outro; à influência do tipo de morte que ocorreu (suicídio, homicídio, morte natural); ao desenvolvimento emocional da pessoa; à duração de uma doença grave; à idade de um paciente ou familiar em processo de morte ou da parente que se perdeu. Hoelter (1979) verificou que o contato direto com a morte tem influência sobre o medo consciente, o medo do processo de morrer e o medo da morte prematura.

Dessa forma, pode-se destacar que o medo da morte se relaciona com uma espécie de sofrimento psíquico, o qual muitas vezes acaba também gerando sofrimento físico, ou criando uma dinâmica incompreensível para quem a vida continua. Na espécie humana, o sofrimento psíquico diante da morte pode ser considerado fisiológico, mas sua duração, intensidade e resolução vão depender muito provavelmente de como a pessoa experimentou a vida (Combinato \& Queiroz, 2006; Brasileiro \& Brasileiro, 2017). 
No que concerne a Tabela 3, a maioria dos docentes respondeu que a temática morte/morrer é abordada na teoria (80,9\%); em atividades práticas/e estágios (61,9\%); que o assunto está descrito no plano de ensino de seus componentes curriculares $(52,3 \%)$ e que discutem a temática durante a prática e/ou o estágio $13(61,9 \%)$. Contudo, 12 (57,1\%) docentes afirmaram que não abordam a temática morte/morrer em seu processo de ensino-aprendizagem.

Os cursos de graduação em Enfermagem no Brasil têm, na última década, incorporado disciplinas que podem dar aporte à compreensão do processo de morrer e da morte; como por exemplo, a inclusão das disciplinas de Psicologia da Saúde, Antropologia da Saúde e Sociologia da Saúde, entre outras (Esquerdo \& Pegoraro, 2010). Em outras palavras, o fenômeno saúde-doença não pode ser ensinado à luz unicamente de instrumentos anatomofisiológicos da medicina, mas deve considerar a visão de mundo dos diferentes segmentos da sociedade, bem como suas crenças e cultura. Significa dizer que nenhum ser humano deve ser estudando e cuidado apenas pelo lado biológico, mas percebido em seu contexto psicosociocultural (Santos, 2012).

Além disso, observa-se que os Planos Pedagógicos de Cursos (PPC) de Bacharelado em Enfermagem vêm sendo reformulados e atualizados, incluindo temáticas vinculadas à Tanatologia e aos Cuidados Paliativos. Estas reformulações se ancoram na Resolução do Conselho Nacional de Saúde (CNS) n 573 de 31 de janeiro de 2018 (Brasil, 2018); à transição epidemiológica que o Brasil vem vivenciando, devido às mudanças nas causas de mortalidade e morbidade secundárias às transformações sociais e econômicas, onde se observa a modificação do perfil de adoecimento da população brasileira, sobretudo de doenças crônicas não transmissíveis, como as doenças cardiovasculares e o câncer (Vasconcelos \& Gomes, 2012; Guimarães et al., 2012); à criação de políticas públicas de saúde que abranjam à Rede de Atenção à Saúde, na perspectiva da Atenção Primária, Secundária e Terciária do Sistema Único de Saúde, dentre elas a Resolução no 41, de 31 de outubro de 2018, que dispõe sobre as diretrizes sobre a organização dos cuidados paliativo (Brasil, 2018) e à atualização do Código de Ética dos Profissionais de Enfermagem (Cofen, 2017), que ressalta no o dever dos profissionais de Enfermagem prestar assistência, no sentido de promover a qualidade de vida no processo do nascer, viver, morrer e luto; e, nos casos de doenças graves incuráveis e terminais com risco iminente de morte, oferecer todos os cuidados paliativos disponíveis para assegurar o conforto físico, psíquico, social e espiritual, respeitando a vontade da pessoa ou de seu representante legal.

Contudo, observa-se que $12(57,1 \%)$ docentes afirmaram que não abordam a temática morte/morrer em seu processo de ensino-aprendizagem. Provavelmente, estes docentes não abordem a temática morte e morrer em suas disciplinas devido à natureza do próprio componente curricular (como por exemplo, Embriologia, Parasitologia, Histologia, Biofísica e Farmacologia, que não apresentam, em suas ementas o processo de cuidar à pessoa nos vários ciclos da vida); à inexperiência na assistência hospitalar por parte do docente; à formação profissional baseada no modelo cartesiano, curativista e hospitalocêntrico e/ou às dificuldades pessoais dos docentes em vivências particulares durante o processo de morte e morrer.

Docentes que se formaram há mais de 10 anos experimentaram um ensino baseado no modelo cartesiano, biomédico, com disciplinas fragmentadas e um cuidado mais voltado para a assistência tecnicista. Por esse motivo, é preciso desconstruir e reconstruir o processo de ensino-aprendizagem que aborde temáticas no entorno da morte e do morrer, levantando questões não restritas à morte em si ou aos procedimentos realizados no corpo pós-óbito, mas destacando a relevância de um processo de cuidar integral, ético e humanizado nos vários ciclos da vida (Bellato et Al., 2007; Carvalho \& Valle, 2006; Pinho \& Barbosa, 2010; Santana et al., 2013; Bandeira et al., 2014; Lima \& Nietsche, 2016; Nunes \& Santos, 2017; Moura et al., 2018).

Além disso, vale ressaltar que a docência é um processo de construção que se faz a partir de mobilização de conhecimentos, valores e percepções que contribuem para a formação da identidade docente. Assim, histórias de família, crenças e vivências educacionais antes da graduação são fatores que influenciam na atuação destes docentes frente ao processo de morte e morrer, sendo inconsistente responsabilizar unicamente a formação acadêmica e às atividades laborais enquanto 
enfermeiros assistenciais (Bellato et al., 2007; Carvalho \& Valle, 2006; Pinho \& Barbosa, 2010; Santana et al., 2013; Bandeira et al., 2014; Lima \& Nietsche, 2016; Nunes \& Santos, 2017; Moura et al., 2018).

Nesta perspectiva, o docente deve passar por um processo de autoconhecimento, para sensibilização, de forma a desenvolver uma visão de terminalidade, de morte e de luto, uma vez que ele é o responsável pela mediação durante o processo de ensino-aprendizagem (Bellato et al., 2007; Carvalho \& Valle, 2006; Pinho \& Barbosa, 2010; Santana et al., 2013; Bandeira et al., 2014; Lima \& Nietsche, 2016; Nunes \& Santos, 2017; Moura et al., 2018).

De acordo com a Tabela 4, as estratégias educativas utilizadas pelos docentes no processo de ensino-aprendizagem sobre a temática morte e morrer envolvem: aula expositiva-dialogada (42,8\%); acompanhamento dos alunos nas atividades teórico-práticas e/ou estágios (23,8\%); trabalhos em grupo e seminários (23,8\%) e exposição de filmes (19\%).

Segundo Lysakowski e Menin (2019), a aula expositiva dialogada é a metodologia mais utilizada pelos professores, cujo objetivo é a explanação do conteúdo que está sendo trabalhado, com a participação ativa dos alunos, em que é possibilitado a troca de conhecimentos.

O trabalho desenvolvido pelos docentes em acompanhar os acadêmicos de enfermagem em hospital de ensino está direcionado à assistência, gestão, formação e pesquisa. O acompanhamento nessas atividades faz com que os acadêmicos desenvolvam a capacidade de lidar com situações distintas e específicas, norteando-os a elaborarem posturas adequadas para cada momento e a utilização de todas as competências desenvolvidas no decorrer da sua vida profissional (Scherer \& Joazeiro, 2015). Dessa forma, os docentes de enfermagem têm a responsabilidade de manter um constante diálogo entre o conhecimento teórico e o prático, estar atento as estratégias de aprendizagem e as reações do acadêmico. Nessa troca de experiências, todos os atores têm a oportunidade de aprender a partir das competências do outro (Souza et al., 2017).

No que concerne à estratégia de utilizar seminários, Perobelli (2018) aponta que o seminário informa, esclarece, transmite um saber, amplia o conhecimento do ouvinte a respeito da temática e aproxima o acadêmico de experiência pedagógica e de liderança.

No que se refere à exposição de filmes como estratégia educativa, Quintino e Ribeiro (2010) ressaltam que é por meio do filme que o discente apreende a temática de maneira sensitiva, pois além das cenas abordarem o conteúdo, o filme evoca emoções e sensações que, posteriormente, suscitam reflexões, partilha de experiências e discussão com os outros estudantes.

Ainda sobre os resultados da Tabela 4, observou-se que os aspectos mais enfatizados pelos docentes durante o processo de ensino-aprendizagem sobre a morte e o morrer foram os cuidados prestados ao paciente e à família durante o processo de morte e morrer $(61,9 \%)$; a doença que causou a morte $(57,1 \%)$ e os sentimentos dos acadêmicos e da equipe de saúde/enfermagem diante da situação de morte do paciente $(57,1 \%)$.

O ensino sobre os cuidados dispensados à família e paciente em processo de morte e morrer é imprescindível, uma vez que o acadêmico de enfermagem precisa conhecer as diretrizes e recomendações no entorno da terminalidade, para que possa praticá-las em campo de estágio. É a partir deste conhecimento que os acadêmicos se nortearão para deter atenção especial às demandas biopsicossociais e espirituais do paciente bem como aos seus familiares e, com isso, promover o melhor cuidado possível (Prado et al., 2017).

Alguns docentes mencionaram que enfatizam a doença que causou a morte do paciente $(57,1 \%)$. No que se refere ao ensino da morte e do morrer, conhecer a doença que causou a morte e/ou a causa mortis é o aspecto de menor importância na perspectiva do processo de cuidar em enfermagem, uma vez que o paciente já foi a óbito. Contudo, numa perspectiva fisiopatológica e/ou epidemiológica, o ensino sobre a doença direciona os acadêmicos sobre as intervenções de enfermagem que podem ser realizadas no âmbito da prevenção, promoção, recuperação de saúde; e numa perspectiva paliativista, o ensino sobre a doença norteia as boas práticas de cuidados paliativos específicos para cada sistema orgânico estudado (Coradazzi, Santana \& Caponero, 2019). 
Outro aspecto enfatizado pelos docentes no processo de ensino-aprendizagem sobre a morte e o morrer é a abordagem dos sentimentos dos acadêmicos e da equipe de saúde/enfermagem diante da situação de morte do paciente $(57,1 \%)$. Sobre este aspecto, defende-se que as escolas de enfermagem devem mudar da preparação dos acadêmicos apenas tecnicamente competentes, para profissionais que sejam capazes de lidar com seus próprios sentimentos e usá-los de modo deliberado e humanamente éticos (Bernieri \& Hirdes, 2007).

Ao proporcionar ao acadêmico um esclarecimento sobre o que realmente é a morte, o docente enriquece o conhecimento do acadêmico acerca de tal processo. Se cada um dos docentes realizasse momentos de reflexão e supervisão após as primeiras experiências dos acadêmicos com a morte, estes se sentiriam mais seguros e preparados para conviver futuramente com tal ocorrência (Bernieri \& Hirdes, 2007).

Desta forma, escutar e acolher os sentimentos de acadêmicos de enfermagem diante de um processo ativo de morte de um paciente é validar os preceitos da ética profissional e da bioética, que preconizam a humanização como pilar fundamental da Enfermagem enquanto profissão (Bernieri \& Hirdes, 2007).

De acordo com a Tabela 5, dez docentes $(47,6 \%)$ responderam que a dimensão espiritual é o cuidado mais realizado pelos seus alunos durante o processo de morte e morrer na assistência de enfermagem.

A consciência/dimensão espiritual e o cuidado espiritual de si mesmo, colegas e pacientes estão no centro de uma prática de enfermagem competente e segura (Keenam \& Macdermot, 2016). A referida dimensão contempla a espiritualidade e a religiosidade. Desta forma, a espiritualidade é inata ao ser humano, constitui-se como recurso para a busca do sentido à vida, não tendo vínculo direto com a religiosidade, e sim, com a própria necessidade de uma pessoa (Arieira et al, 2017). Ela pode ser utilizada como um instrumento de adaptação às circunstâncias adversas da vida (Soares \& Amorim, 2015).

O enfermeiro, ao assistir o paciente nas 24 horas, precisa ter embasamento cientifico para atuar mediante visão holística, contemplando a dimensão espiritual que, ao ser abordada, contribui para satisfazer as necessidades espirituais do indivíduo. O olhar holístico é fundamental, pois quando a espiritualidade é valorizada, as experiências pessoais de dor e sofrimento podem tornar-se suportáveis (Sampaio \& Siqueira, 2016).

Nesse contexto, o cuidado espiritual de enfermagem é aquele que reconhece e atende às necessidades do espírito em momentos de doença e tristeza, podendo também incluir necessidade por sentido, suporte de fé, oração ou simplesmente uma escuta sensível. Este cuidado começa encorajando o contato humano em relações de compaixão e se move para onde as necessidades requerem (Miquelatto et al., 2017). O enfermeiro desenvolve a assistência baseada na Sistematização da Assistência de Enfermagem (SAE), cujo ponto de partida é a coleta de dados do paciente. Nesse primeiro momento, durante essa coleta, a enfermeira detecta as necessidades da pessoa, razão pela qual é capaz de abordar a avaliação da espiritualidade como parte fundamental do ser humano sujeito ao cuidado (Giske \& Conne, 2015).

De acordo com as Diretrizes Curriculares Nacionais do Curso de Graduação em Enfermagem (DCNs) e Recomendações do Conselho Nacional de Saúde à proposta das Diretrizes Curriculares Nacionais (DCN) para os cursos de graduação em Enfermagem, o perfil do egresso deve se pautar numa formação generalista, humanista, crítica e reflexiva capaz de conhecer e intervir sobre os problemas e situações de saúde-doença, identificando os aspectos biopsicossociais e espirituais e seus determinantes, relacionando a sua práxis ao senso de responsabilidade social e compromisso com a cidadania, como representante social da saúde integral do ser humano, o que inclui o autocuidado físico e psíquico, bem como a busca pelo bem-estar da população (Brasil, 2001; Brasil, 2018).

Desse modo, ressalta-se a importância da abordagem do ensino do processo de morte e morrer de forma contínua ao longo do percurso acadêmico, não focalizando em uma disciplina específica, mas na integração de componentes curriculares diversos, bem como com uma articulação da temática com ações extensionistas, realização de pesquisas e criação de grupos de 
estudos voltados para a temática (Bellato et al., 2007; Carvalho \& Valle, 2006; Pinho \& Barbosa, 2010; Santana et al., 2013; Bandeira et al., 2014; Lima \& Nietsche, 2016; Nunes \& Santos, 2017; Moura et al., 2018).

\section{Considerações Finais}

O presente estudo investigou os caminhos utilizados pelos docentes de enfermagem sobre a temática morte e morrer durante a formação acadêmica de um curso de Bacharelado em Enfermagem em um campus universitário do interior da Paraíba e apontou que a maioria dos docentes são mulheres, com idade média de 38 anos, formados há mais de 10 anos e com experiência na assistência e na docência.

Os resultados mostraram que a maioria dos docentes de enfermagem incluem a temática morte e morrer em seus planos de ensino; apresentam o assunto nas aulas teóricas e discutem o tema nas atividades teórico-práticas e/ou em estágios supervisionados. Além disso, o estudo também apontou que a maioria dos docentes utilizam aulas expositivas-dialogadas, expõem filmes e realizam seminários como estratégias metodológicas de ensino para debater a temática morte e morrer. Contudo, parte significante dos docentes não abordam o tema nos seus componentes curriculares teóricos e práticos.

O estudo destacou que ao presenciar um processo de morte e morrer nas atividades teórico-práticos e/ou em estágios supervisionados, os docentes explanam enfaticamente os cuidados dispensados ao paciente e à família, discutem sobre a doença que causou a morte e acolhem os sentimentos dos acadêmicos e da equipe de enfermagem. Em relação à sistematização da assistência de enfermagem, os docentes referiram que os acadêmicos de enfermagem priorizam o cuidado espiritual quando presenciam o processo ativo de morte de um paciente nas atividades teórico-prático e/ou estágio supervisionado.

No que se refere às emoções e sentimentos dos docentes em relação à temática, os resultados apontaram que a maioria aceita a morte como etapa natural da vida, contudo, outra parcela significante de docentes mencionou que a morte suscitava medo e pavor.

De forma geral, pode-se observar que a maioria dos docentes de enfermagem abordam a temática morte e morrer na formação acadêmica, de forma transversal, uma vez que a grade curricular contempla disciplinas que abrangem a saúde coletiva, a organização dos serviços de enfermagem e cuidados de enfermagem nos processos de nascer, viver, morrer e luto com vistas à qualidade de vida e dignidade humana.

Nesse sentido, vale ressaltar que o Projeto Pedagógico do Curso de Bacharelado em Enfermagem do Centro de Educação e Saúde da Universidade Federal de Campina Grande foi alterado em 2015, possibilitando o aumento da carga horária teórica e prática dos componentes curriculares profissionalizantes obrigatórios e a oferta de componentes curriculares optativos, tais como Cuidados Paliativos e Enfermagem.

Os resultados deste estudo mostraram uma modificação do processo de ensino-aprendizagem na formação acadêmica de enfermagem no tocante à temática morte, contudo, ainda se observou um contingente de docentes que não abordaram a temática morte e morrer em seus componentes curriculares, seja porque o conteúdo não se adéque à realidade do plano de curso de sua disciplina, seja pela inexperiência na área assistencial ou seja pelos sentimentos e emoções evocados ao refletirem sobre a morte.

Por isso, se faz necessário conversar sobre a morte, trazê-la para dentro da academia, abrindo espaços para educação para morte, seja no ensino, na pesquisa ou na extensão. O importante é trazer à tona reflexões, discussões e ações que aproximem os acadêmicos do fenômeno morte do mesmo modo que experienciam o fenômeno do nascimento, desenvolvimento e envelhecimento do ser humano.

É a partir do processo de ensino-aprendizagem sobre os vários ciclos da vida que acadêmicos aprendem e desenvolvem habilidades para o cuidar. Cuidar da vida implica em cuidar da morte, pois a responsabilidade profissional é com 
o amparo da vida: daquele que está por nascer e daquele que está por morrer. Portanto, para melhor cuidar de alguém que está morrendo, é preciso refletir sobre a morte: a do paciente, a do acadêmico e a do docente.

Um limite deste estudo foi o mesmo ter sido realizado em apenas um local, em momento e cenário historicamente datados, em um contexto cultural particular, outro foi que focou o processo de ensino-aprendizagem acerca da morte e do morrer na perspectiva de docentes de enfermagem. Deste modo, fazem-se necessárias mais pesquisas nesta área, em diferentes cenários e com ênfase na atuação do acadêmico de enfermagem frente ao processo ativo de morte de pacientes atendidos nos mais diversos cenários da rede de atenção à saúde.

\section{Referências}

Andrade, C. B. (2015). O trabalho de cuidar e educar: gênero, saber e poder. Appris. Paraná.

Arieira I. C. O, Thofehrn M. B, Milbrath V. M, Schwonke, C. R. G. B., \& Cardoso D. H, Fripp J. C. (2017). The meaning of spirituality in the transience of life. Esc Anna Nery, 21(1). https://www.scielo.br/j/ean/a/F5n46JqtVcqbG8PvW68FqML/?lang=pt\&format=pdf.

Bandeira, D, Cogo, S. B, Hildebrandt, L. M., \& Badke, M. R. (2014). A morte e o morrer no processo de formação de enfermeiros sob a ótica de docentes de enfermagem. Texto Contexto Enferm, 23(2), 400-7. http://www.scielo.br/pdf/tce/v23n2/pt_0104-0707-tce-23-02-00400.pdf.

Bellato, R, Araújo, A. P, Ferreira, H. F., \& Rodrigues, P. F. (2007). A abordagem do processo do morrer e da morte feita por docentes em um curso de graduação em enfermagem. Acta Paulista de Enfermagem, 20(3), 255-263. https://www.scielo.br/pdf/ape/v20n3/a03v20n3.pdf.

Bernieri, J., \& Hirdes, A. (2007). O preparo dos acadêmicos de enfermagem brasileiros para vivenciarem o processo de morte e morrer. Texto Contexto, 16(1), 89-96. https://www.scielo.br/j/tce/a/KYDRwZ7V3SFqTkh7kg5CPzM/?lang=pt.

Brasil. (2001). Ministério da Educação. Diretrizes Curriculares Nacionais dos Cursos de Graduação em Enfermagem. Medicina e Nutrição. Brasília. Ministério da Educação. Disponível em: http://portal.mec.gov.br/dmdocuments/ces1133.pdf.

Brasil. (2012). Ministério da Saúde. Resolução 466/2012 do Conselho Nacional de Saúde do Ministério da Saúde. Dispõe sobre a aprovação de diretrizes e normas regulamentadoras de pesquisas envolvendo seres humanos.

Brasil. (2018). Ministério da Saúde (BR). Gabinete do Ministro. Comissão Intergestores Tripartite. Resolução nº 41 de 31 de outubro de 2018 . Dispõe sobre as diretrizes para a organização dos cuidados paliativos, à luz dos cuidados continuados integrados, no âmbito do Sistema Único de Saúde. Diário Oficial da União. Brasília. Edição 225. Seção 1. Página 276.

Barbosa, L. N. F., Francisco, A. L., \& Efken, K. H. (2018). Morte e Vida: a dialética humana. Aletheia, (28), 32-44. http://pepsic.bvsalud.org/pdf/alethei a/n28/n28a04.pdf.

Brasil. Ministério da Saúde. Conselho Nacional de Saúde. Resolução N ${ }^{o} 573$ de 31 de janeiro de 2018. Recomendações do Conselho Nacional de Saúde à proposta de Diretrizes Curriculares Nacionais (DCN) do curso de graduação Bacharelado em Enfermagem. Disponível em: https://www.in.gov.br/materia//asset_publisher/Kujrw0TZC2Mb/content/id/48743098/do1-2018-11-06-resolucao-n-573-de-31-de-janeiro-de-2018-48742847

Brasileiro, M., \& Brasileiro, J. E. (2017). O medo da morte enquanto mal: uma reflexão para a prática da enfermagem. Revista de Ciências Médicas, 26(2), 7792, 2017. DOI:10.24220/2318-0897V26N2A3582.

Carneiro, A. C. M. O., \& Andrade, A. C. R. (2019). O despertar para o cuidado na morte. Com Ciências Saúde, (29), 54-7. http://www.escs.edu.br/revistaccs/index.php/comunicacaoemcienciasdasaude/article/view/174/131.

Carvalho, M. D. B., \& Valle, E. R. M. (2016). Vivência da morte com o aluno na prática educativa. Ciência, Cuidado e Saúde, Maringá, (5), 26-32. http://periodicos.uem.br/ojs/index.php/CiencCuidSaude/article/view/5149/3335.

Collet, L. J., \& Lester, D. (1969). O medo da morte e o medo de morrer. The journal of Psychology, 72(2), 179-181. https://doi.org/10.1080/0022398 0.19 69.10543496.

Conte, H. R., Weiner, M. B., \& Plutchik, R. (1982). Medindo a ansiedade da morte: Aspectos conceituais, psicométricos e analíticos fatoriais. Journal of Personality and Social Psychology, 43(4), 775-785. https://doi.org/10.1037/0022-3514.43.4.775.

Combinato, D. S., \& Queiroz, M. S. (2006). Morte: uma visão psicossocial. Estudos de Psicologia (Natal), (11), 209-216. https://www.scielo.br/j/epsic/a/PfSWjx6JP7NQBWhcMBXmnyq/?lang=pt\&format=pdf

Coradazzi, A. L., Santana, M. T. E. A., \& Caponero, R. (2019). Cuidados paliativos: diretrizes para melhores práticas. MG Editores.

Esquerdo, A. F., \& Pegoraro, R. F. (2010). Contribuições da psicologia para a formação do técnico em enfermagem: concepções dos alunos. Rev. Psicologia em Estudo, 15(2), 255-264. https://www.scielo.br/j/pe/a/S3g6Zq9vLMqt5sTFCXzBLCp/?lang=pt\&format=pdf.

Giske, T., \& Cone P. H. (2015). Discerning the healing path-how nurses assist patient spirituality in diverse health care settings. J Clin Nurs, 24(19-20), 292635, 2015. 10.1111/ jocn.12907. 
Guimarães, R. M., Muzi, C. D., Teixeira, M. P., \& Pinheiro, S. S. (2016). A transição da mortalidade por cânceres no Brasil e a tomada de decisões estratégicas nas políticas públicas de saúde da mulher. Rev Pol Pub, 20(1), 33-50. em:http://www.periodicoseletronicos.ufma.br/ index.php/rppublica/article/view/5035/3077.

Hoelter, J. W. (1979). Tratamento multidimensional do medo da morte. Journal of Consulting and Clinical Psychology, 47(5), 996-999. https://doi.org/10.1037/0022-006X.47.5.996.

Keenan P. M., \& MacDermott, C. (2016). Prayer and Religion: Irish nurses caring for an intellectually disabled child who has died. Religions, 7(12), 148. http://www.mdpi.com/2077-1444/7/12/148.

Kovács, M. J. (2004). Educação para a morte: temas e reflexões. (2a ed.). Ediouro.

Kovács, M. J. (2008). Morte e Existência Humana: caminhos de cuidados e possibilidades de intervenção. Guanabara-Koogan.

Lima, M. G. R., \& Nietsche, E. A. (2016). Ensino da morte por docentes enfermeiros: desafio no processo de formação acadêmica. Rev Rene. 17(4), 512-9. http://www.periodicos.ufc.br/rene/article/view/4946/3647.

Lysakowski, S., \& Menin, G. E. (2019). Utilização de simulação clínica no ensino sobre terminalidade da vida na Enfermagem: relato de experiência. Rev Docência Do Ensino Superior, (9), 1-14. https://doi.org/10.35699/2237-5864.2019.2559.

Miquelatto, M., Silva, L., Figueira, C. B., Santos, M. R., Szilit, R., \& Ichikawa, C. R. F. (2017). Espiritualidade de famílias com um ente querido em situação final de vida. Rev Cuidarte, 8(2), 1016-27. https://www.revistacuidarte.org/index.php/cuidarte/ article/view/391/798.

Moura, L. V. C., Passos, E. C. S., Santos, R. M. M., Rosa, D. O. S., \& Nascimento, S. C. L. (2018). Ensino da tanatologia nos cursos de graduação em enfermagem. Rev Baiana Enferm, (32), e20888. https://portalseer.ufba.br/index.php/enfermagem/article/view/20888/15703.

Nunes, E. C. D. A., \& Santos, A. A. (2017). Desafios de ensino-aprendizagem da Enfermagem para o cuidado frente ao morrer humano-percepções docentes. Escola Anna Nery, 21(4), e20170091. https://www.scielo.br/scielo.php?pid=S1414-81452017000400235\&script=sci_arttext\&tlng=pt.

Nunes, F. N., Araújo, K. M., \& Silva, L. D. C. (2016). As evidências sobre o impacto psicossocial de professores de enfermagem frente à morte. Rev Interd, 9(4), 165-72. https://revistainterdisciplinar.uninovafapi.edu.br/index.php/revinter/article/view/545.

Pereira, A. S., Shitsuka, D. M., Parreira, F. J., \& Shitsuka, R. (2018). Metodologia da pesquisa científica.

Pereira, I. B., \& Ramos, M. N. (2006). Educação profissional em saúde. Fiocruz.

Perobelli, F. S., Araújo Júnior, I. F., \& Castro, L. S. (2018). As dimensões espaciais da cadeia produtiva do leite em Minas Gerais. Nova Economia, 28(1). https://revistas.face.ufmg.br/index.php/novaeconomia/article/view/4789.

Pinho, L. M. O., \& M. A. (2010). A relação docente-acadêmico no enfrentamento do morrer. Rev Escola Enferm USP, 44(1), 107-112. https://www.scielo.br/pdf/reeusp/v44n1/a15v44n1.pdf. Acesso em: 19 maio 2020.

Prado, R. T., Leite, J. L., Castro, E. A. B., Silva, L, J., \& Silva, I. R. (2018). Desvelando os cuidados aos pacientes em processo de morte/morrer e às suas famílias. Rev Gaúcha Enferm, (39), 2017-0111. http://www.scielo.br/pdf/rgenf/v39/1983-1447-rgenf-39-e2017-0111.pdf.

Praxedes, A. M., Araújo, J. L., \& Nascimento, E. G. C. (2018). A morte e o morrer no processo de formação do enfermeiro. Psic Saúde e Doenças. 9(12), 36976. http://www.scielo.br/pdf/tce/v23n2/pt_0104-0707-tce-23-02-00400.pdf.

Quintino, C. P., Ribeiro, K. D. F. (2010). A Utilização de filmes no processo de ensino aprendizagem de Química no Ensino Médio [Apresentação de painel]. XV Encontro Nacional de Ensino de Química (XV ENEQ). Brasília, DF, Brasil. http://www.sbq.org.br/eneq/xv/resumos/R0472-1.pdf.

Sampaio, A. D., \& Siqueira, H. C. H. (2016). Influência da espiritualidade no tratamento do usuário oncológico: olhar da enfermagem. Ensaios Cienc Biol Agrar Saúde, 20(3), 151-8. http://pgsskroton.com.br/seer/index.php/ensaioeciencia/article/view/4433 7.

Santana, J. C. B., Santos, A. V., Silva, B. R., Oliveira, D. C. A., Caminha, E. M., Peres, F. S., \& Andrade, C. C. D. (2013). Docentes de enfermagem e terminalidade em condições dignas. Rev Bioética, 21(2), 298-307. https://www.scielo.br/pdf/bioet/v21n2/a13v21n2.pdf.

Santos, A. C. B., Silva, A. F., Sampaio, D. L., Sena, L. X., Gomes, V. R., \& Azevedo, V. L. (2012). Antropologia da saúde e da doença: contribuições para a construção de novas práticas em saúde. Rev Nufen, 4(2), 11-21. http://pepsic.bvsalud.org/scielo.php?script=sci_arttext\&pid=S2175-25912012000200003.

Scherer, M. D. A., \& Joazeiro, E. M. G. (2015). Trabalho coletivo em hospitais de ensino: desafios e reservas de alternativas. Trabalho e Saúde: cenários, impasses e alternativas no contexto brasileiro. Opção Editora.

Silva, C. L. N., \& Melo, T. C. L. (2018). “Quem de novo não morre, de velho não escapa”: uma pesquisa bibliográfica acerca das publicações em Tanatologia no período de 2012 a 2017 no Brasil. Cad Grad Psicol 4(3), 173-86. https://periodicos.set.edu.br/index.php/fitshumanas/article/view/5228.

Soares, A. S., \& Amorim, M. I. (2015). Qualidade de vida e espiritualidade em pessoas idosas institucionalizadas. Rev Port Enferm Saude Mental, (2), 45-51. http://www.scielo.mec.pt/pdf/rpesm/nspe2/nspe2a08.pdf

Souza, D. J., Faria, M. F., Cardoso, R. J., \& Contrim, D. (2017). Estágio curricular supervisionado sob a óptica dos enfermeiros supervisores. Rev Enferm Atenção Saúde, 6(1), 39-51. http://seer.uftm.edu.br/revistaeletronica/index.php/enfer/article/view/1677.

Vasconcelos, A. M. N., \& Gomes, M. M. F. (2012). Transição demográfica: a experiência brasileira. Epidemiol. Serv. Saúde, 21(4), 539-548. http://scielo.iec.gov.br/pdf/ess/v21n4/v21n4a03.pdf. 